[6] V. Shapiro, Topics in Fourier and geometric analysis, Mem. Amer. Math. Soc. 39 (1961), pp. 1-100.

[7] E. C. Titchmarsh, Eigenfunotion expansions associated with second order ifferential equations, part II, second edition, Oxford 1958.

[8] A. Zygmund, Trigonometrical series, 2 volumes, Cambridge 1958.

INSTITUTE OF MATHEMATICS,

LUND, SWEDEN

\section{Über die Methode des , schnellsten Falles" für das Minimumproblem von Funktionalen in Hilbertschen Räumen}

von

T. LE⿱亡AA ŃSKI (Warszawa)

Auf einem reellen Hilbertschen Raum sei ein reelles, differenzierbares Funktional $\Phi(x)$ erklärt. Unter einem Gradienten von $\Phi$ versteht man bekanntlich dasjenige Element: grad $\Phi(x)$, welches die Gleichung $\Phi^{\prime}(x, h)$ $=(\operatorname{grad} \Phi(x), h)$ identisch in $h \in H$ erfüllt. Die in dieser Note betrachtete Methode besteht in folgendem: man nimmt an, daß die Gleichung

$$
\frac{d x(t)}{d t}=-\operatorname{grad} \Phi(x(t))
$$

eine Lösung für alle $t \geqslant 0$ besitzt; dann konvergiert unter gewissen Voraussetzungen $\Phi(x(t))$ gegen sein Minimumwert inf $\Phi(x)$, oder auch $x(t)$ gegen Element $x^{*}$, mit $\Phi\left(x^{*}\right)=\inf \Phi(x)$. Auch der Fall, wenn die Gleichung (I) nur annäherungsweise erfüllt ist, wird behandelt.

Die Idee dieser Methode rührt schon vom A. Cauchy her, und wurde von anderen Verfassern verallgemeinert (s. [1] und [2]).

SATz 1. Sei $\Phi(x)$ ein auf $H$ erklärtes, konvexes, stetiges von unten beschränktes Funktional, dessen Gradient stetig ist. Die abstrakte Funktion $x(t)$ erfülle die Gleichung (I). Dann gilt:

$$
\lim \Phi(x(t))=\inf \Phi(x) .
$$

Beweis erfolgt in folgenden Schritten:

LEMMA 1. Erfüllen die Elemente $x_{1}, x_{2}$ die Ungleichung $\Phi\left(x_{2}\right)<\Phi\left(x_{1}\right)$, so gilt:

$$
\left\|\Phi\left(x_{1}\right)-\Phi\left(x_{2}\right)\right\| \leqslant\left\|\operatorname{grad} \Phi\left(x_{1}\right)\right\| \cdot\left\|x_{1}-x_{2}\right\| .
$$

Aus der Konvexität von $\Phi$ ergibt sich nämlich also:

$$
\Phi\left(x_{1}+\varepsilon\left(x_{2}-x_{1}\right)\right) \leqslant \Phi\left(x_{1}\right)+\varepsilon\left[\Phi\left(x_{2}\right)-\Phi\left(x_{1}\right)\right] \quad \text { für } \quad 0<\varepsilon<1
$$

$$
\frac{1}{\varepsilon}\left\{\Phi\left(x_{1}+\varepsilon\left(x_{2}-x_{1}\right)\right)-\Phi\left(x_{1}\right)\right\} \leqslant \Phi\left(x_{2}\right)-\Phi\left(x_{1}\right)
$$


und der Grenzübergang $\varepsilon \rightarrow 0$ liefert

d.h.

$$
\Phi^{\prime}\left(x_{1}, x_{2}-x_{1}\right)=\left(\operatorname{grad} \Phi\left(x_{1}\right), x_{2}-x\right)_{1} \leqslant \Phi\left(x_{2}\right)-\Phi\left(x_{1}\right),
$$

$$
\Phi\left(x_{1}\right)-\Phi\left(x_{2}\right) \leqslant\left(\operatorname{grad} \Phi\left(x_{1}\right), x_{1}-x_{2}\right) \leqslant\left\|\operatorname{grad} \Phi\left(x_{1}\right)\right\| \cdot\left\|x_{1}-x_{2}\right\| .
$$

LEMna 2. Sei $\bar{z}$ ein fixiertes Element. Erfüllt die abstrakte Funktion $x(t)$ die Ungleichung $\Phi(x(t))>\Phi(\bar{z})$ für $t_{1} \leqslant t \leqslant t_{2}$, so gilt

$$
\frac{d}{d t}\|x(t)-\bar{z}\|^{2} \leqslant 0 \quad \text { für } \quad t_{1} \leqslant t \leqslant t_{2} .
$$

In der Tat, wir haben

$$
\begin{aligned}
\frac{d}{d t}\|x(t)-\bar{z}\|^{2} & =(x(t)-\bar{z}, d x(t) / d t) \\
& =(x(t)-\bar{z},-\operatorname{grad} \Phi(x(t)))=\Phi^{\prime}(x(t), \bar{z}-x(t)) \\
& =\lim _{\varepsilon \rightarrow 0} \frac{1}{\varepsilon}\{\Phi(x(t)+\varepsilon(\bar{z}-x(t)))-\Phi(x(t))\} \\
& =\lim _{\varepsilon \rightarrow 0} \frac{1}{\varepsilon}\{\Phi((1-\varepsilon) x(t)+\varepsilon \bar{z})-\Phi(x(t))\} \leqslant 0
\end{aligned}
$$

für kleine Werte von $\varepsilon$, wegen der Konvexität von $\Phi$ und der Voraussetzung $\Phi(\bar{z})<\Phi(x(t))$.

Beweis des Satzes 1 . Nehmen wir im Gegenteil an, daß es ein Element $\bar{z}$ und eine Zahl $\varepsilon>0$ gibt, die die Ungleichung $\varepsilon+\Phi(\bar{z}) \leqslant$ $\leqslant \Phi(x(t))$ für alle $t \geqslant 0$ erfüllen, insbesondere also für $0<t_{1} \leqslant t \leqslant t_{2}$, wobei $t_{1}<t_{2}$ zwei fixierte Zahlen bedeuten. Wenden wir nun die Lemmata 1 und 2 entsprechend an, so erhalten wir:

$$
\begin{aligned}
\{\Phi(x(t))-\Phi(\bar{z})\} & =\langle\operatorname{grad} \Phi(x(t)), x(t)) \\
& =-\|\operatorname{grad} \Phi(x(t))\|^{2} \leqslant-\{\Phi(x(t))-\Phi(\bar{z})\}^{2} \cdot\|x(t)-\bar{z}\|^{-2} \\
& \leqslant-\{\Phi(x(t))-\Phi(\bar{z})\}^{2} \cdot\left\|x\left(t_{1}\right)-\bar{z}\right\|^{-2} \leqslant 0 .
\end{aligned}
$$

Die damit erhaltene Ungleichung

$$
\frac{d\{\Phi(x(t))-\Phi(\bar{z})\} / d t}{\{\Phi(x(t))-\Phi(\bar{z})\}^{2}} \leqslant \frac{-1}{\left\|x\left(t_{1}\right)-\bar{z}\right\|^{2}}
$$

integrieren wir von $t_{2}$ bis $t_{1}$ und wir bekommen:

$$
\begin{aligned}
\Phi\left(x\left(t_{2}\right)\right)-\Phi(\bar{z}) & \leqslant\left\{\left[\Phi\left(x\left(t_{1}\right)\right)-\Phi(\bar{z})\right]^{-1}+\left(t_{2}-t_{1}\right)\left\|x\left(t_{1}\right)-\bar{z}\right\|^{-1}\right\}^{-1} \\
& \leqslant\left\|x\left(t_{1}\right)-\bar{z}\right\| \cdot\left(t_{2}-t_{1}\right)^{-1}
\end{aligned}
$$

Lassen wir nun $t_{2}$ gegen $+\infty$ streben, so erhalten wir einen Widerspruch mit der Annahme $\Phi(\bar{z})+\varepsilon \leqslant \Phi(x(t))(t \geqslant 0)$. Es ist also inf $\Phi(x(t))=$ $=[\inf \Phi(y), y \in H]$, und, da $d \Phi(x(t)) / d t \leqslant 0$ gilt, auch $\lim _{t \rightarrow \infty} \Phi(x(t))=\inf \Phi(x)$, w.z.b.w.

SATz 2. Zu den Voraussetzungen des Satzes 1 geben wir noch zu, daß das Funktional $\Phi$ quadratisch ist, und daß der Minimumwert von $\Phi$ in gewissem Punkte $\bar{x}$ erreicht wird, d.h. $\Phi(\bar{x})=\inf \Phi$. Bei diesen zusätzlichen Voraussetzungen konvergiert die die Gleichung (I) erfüllende abstrakte Funktion $x(t)$ stark gegen ein Element $x^{*}$, und $\Phi\left(x^{*}\right)=\inf \Phi$.

Beweis. Wegen der Voraussetzung über $\Phi$ läßt sich $\Phi(x)$ in der Gestalt $\Phi(x)=\frac{1}{2}(A x, x)+(a, x)$ darstellen, wobei $a$-ein fixiertes Element, $A$ - eine lineare, beschränkte, symmetrische nichtnegative Operation bedeuten. Die Lösung der Gleichung $d x(t) / d t=-\operatorname{grad} \Phi(x(t))=$ $-(A x(t)-a)$ hat in diesem Fall die Gestalt

$$
x(t)=b-\int_{0}^{t} \exp (-s A)(A b-a) d s, \quad \text { mit } \quad b=x(0),
$$

wie es leicht nachzuprüfen ist. Laut der Voraussetzung gibt es ein $\bar{x}$, für welches die Gleichung $\Phi(\bar{x})=\inf \Phi$, also auch $0=\operatorname{grad} \Phi(\bar{x})=A(\bar{x})-a$ gilt. Wir können also (1), indem wir mit $M$ - die obere Schranke, mit $E_{\lambda}$ - die spektrale Familie von $A$ bezeichen, auf folgende Weise umschreiben:

$$
\begin{aligned}
x(t) & =b-\int_{0}^{t} \exp (-s A)(A(b-\bar{x})) d s \\
& =b-\left\{\int_{0}^{t} \exp (-s A) A d s\right\}(b-\bar{x}) \\
& =b-\left\{\int_{0}^{M}\left[\int_{0}^{t} \exp (-s \lambda) \lambda d s\right] d E_{\lambda}\right\}(b-\bar{x}) \\
& =b-\left[\int_{0}^{M}\left(1-\exp (-t \lambda) d E_{\lambda}\right](b-\bar{x}) .\right.
\end{aligned}
$$

Die Operationen $U_{t}=\int_{0}^{M}[1-\exp (-t \lambda)] d E_{\lambda}$ sind symmetrisch, positiv, und von oben mit der Identität beschränkt; sie stellen außerdem, als Funktionen des Parameters $t$ verstanden, eine nichtabnehmende Operationenfunktion dar (s. [3], No 106,107 S. 267 ff.) $U_{t}$ konvergiert also mit $t \rightarrow \infty$, im starken Sinne gegen gewisse symmetrische, beschränkte Operation $U_{\infty}$ (s. [3], $\mathrm{N}^{0} 104$, S. 261). Insbesondere haben wir:

$$
x(t)=b-U_{t}(b-\bar{x}) \rightarrow x^{*}=b-U_{\infty}(b-\bar{x}) .
$$


Da aber, laut Satz 1, $\Phi(x(t)) \rightarrow \inf \Phi$ gilt,

$$
\Phi\left(x^{*}\right)=\inf \Phi, \quad \text { w.z.b.w. }
$$

Wir geben nun eine Anwendung des Satzes 2 , an. Sei $B$ lineare, beschränkte, nicht notwendig symmetrische Operation, $y_{0}$ - fixiertes Element, die Menge $Z=\left[x: B x-y_{0}=0\right]$ und sei $0 \neq Z \neq H$. $\quad b \notin Z$. Wir stellen uns die Aufgabe, die orthogonale Projektion von $b$ auf die Menge $Z \mathrm{zu}$ finden. $Z u$ diesem Zwecke legen wir

$$
\Phi(x)=\left\|B x-y_{0}\right\|^{2}, \quad A=2 B^{*} B, \quad \bar{x}=2 B^{*}\left(y_{0}\right)
$$

fest. Es gilt dann:

$$
\operatorname{grad} \Phi(x)=2 B^{*}\left(B x-y_{0}\right)=A x-\bar{x} .
$$

Die abstrakte Funktion

$$
x(t)=b-\int_{0}^{t} \exp (-s A)(A b-\bar{x}) d s
$$

erfüllt ersichtlich die Gleichungen $d x(t) / d t=-A(x(t)-b)=-\operatorname{grad} \Phi(x(t))$ $x(0)=b$. Da es laut Voraussetzung $0 \neq Z$ Elemente $x$ gibt, die die Gleichungen $B(x)-y_{0}=0 \equiv \Phi(x)=\inf \Phi$ erfüllen, und da $\Phi(x)$ quadratisch ist, konvergiert $x(t)$ laut Satz 2 gegen ein Element $x^{*}$, so daß die Gleichung $B\left(x^{*}\right)-y_{0} \equiv\left[\Phi\left(x^{*}\right)=0=\inf \Phi\right]$ gilt.

Wir werden zeigen, daß $x^{*}=\operatorname{proj}(b) \mid Z$ - die orthogonale Projektion von $b$ auf die Menge $Z$ ist, d.h., daß die Gleichung $\left(b-x^{*}\right.$, $\left.x_{1}-x_{2}\right)=0$ für jedes Paar $x_{1}, x_{2}$ aus $Z$ gilt. Wir haben aber

$$
\begin{gathered}
\left(b-x(0), x_{1}-x_{2}\right)=\left(b-b, x_{1}-x_{2}\right)=0, \\
\left.\frac{d}{d t}\left(b-x(t), x_{1}-x_{2}\right)=-\left(x(t)-b, x_{1}-x_{2}\right)\right) \\
=2\left(B^{*}\left(B(x(t))-y_{0}\right), x_{1}-x_{2}\right)=2\left((B x(t))-y_{0}, B(x)-B\left(x_{2}\right)\right) \\
=2\left(B(x(t))-y_{0}, y_{0}-y_{0}\right)=0 .
\end{gathered}
$$

Daraus folgt $(x(t)-b, x-x)=0$ für alle $t \geqslant 0$, also

$$
\left(x^{*}-b, x_{1}-x_{2}\right)=\lim \left(x(t)-b, x_{1}-x_{2}\right)=0 .
$$

Die bewiesene Tatsache kann man in folgende Formel auffassen:

$$
\operatorname{proj}(b) /\left[x: B(x)-y_{0}=0\right]=b-\int_{0}^{\infty} \exp (-s A)(A b-\bar{x}) d s,
$$

wobei $A=2 B^{*} B, \bar{x}=2 B^{*}\left(y_{0}\right)$.
Wir legen jetzt eine Modifizierung der Methode des „schnellsten Falles" vor, die eine Methode des ,genügend schnellen Falles" genannt werden darf. Der Unterschied besteht darin, daß in der neuen Methode die Gleichung $d x(t) / d t=-\operatorname{grad} \Phi(x(t))$ schon nicht mehr erfüllt werden muß; statt deren muß die Funktion $x(t)$ die Eigensehaft haben, daß ihre Ableitung $d x(t) / d t$ mit dem Wektor $-\operatorname{grad} \Phi(x(t))$ einen nicht zu großen Winkel bildet. Die Konvexität von $\Phi$ wird nicht mehr verlangt.

SATZ 3. Sei $\Phi(x)$ ein stetiges, von unten beschränktes Funktional, mit stetigem Gradienten $F(x)$; sei $x(t)$ eine stetige, stückweise differenzierbare abstrakte Funktion, die zusammen mit dem Gradienten $F^{\prime}(x)$ die folgenden Voraussetzungen erfüllt:

$$
\begin{aligned}
(\dot{x}(t), F(x(t))) & \leqslant-a\|\dot{x}(t)\| \cdot\|F(x(t))\|, \\
\beta\|\dot{x}(t)\| \leqslant & \|F(x(t))\| \leqslant \gamma\|\dot{x}(t)\|, \\
& 0<a<1,0<\beta<\gamma, \dot{x}(t)=d / d t x(t),
\end{aligned}
$$

(iii) es ist eine reelle Funktion $f(s)$ vorhanden, $f(0)=0, f^{\prime}(s)>0$, die die folgenden Ungleichungen erfüllt:

$$
\int_{0}^{\Phi(x(0))-\inf \Phi} \frac{d s}{f(s)}<+\infty, \quad\|F(x(t))\| \geqslant f[\Phi(x(t))-\inf \Phi] .
$$

Dann konvergiert $x(t)(t \rightarrow \infty)$ gegen ein $x^{*}$, für welches die Gleichungen $F\left(x^{*}\right)=0, \Phi\left(x^{*}\right)=\inf \Phi$ gelten .

Beweis. Bezeichnen wir $m=\inf \Phi$; wir haben

$$
\begin{aligned}
-\frac{d}{d t}[\Phi(x(t))-m] & =-(\dot{x}(t), F(x(t))) \\
& \geqslant a\|\dot{x}(t)\| \cdot\|F(x(t))\| \geqslant \alpha\|\dot{x}(t)\| \cdot\{f[\Phi(x(t))-m]\}
\end{aligned}
$$

also

$$
\|\dot{x}(t)\| \leqslant \frac{-1}{a} \cdot \frac{d}{d t}[\Phi(x(t))-m]\{f(\Phi(x(t))-m)\}^{-1} .
$$

Für jedes Zahlenpaar $0 \leqslant t_{1} \leqslant t_{2}$ gilt also

$$
\begin{aligned}
& \left\|x\left(t_{2}\right)-x\left(t_{1}\right)\right\| \leqslant \int_{t_{1}}^{t_{2}}\left\|\frac{d}{d t} x(t)\right\| d t
\end{aligned}
$$

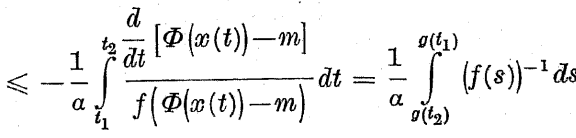


mit $g(t) \stackrel{\text { Df }}{=} \Phi(x(t))-m . g(t)$ ist aber, infolge (3), eine nichtwachsende Funktion mit positiven Werten; daher muß $g(t)$ mit $t \rightarrow \infty$ einen Limeswert haben. Folglich konvergiert das letztgeschriebene Integral gegen Null, mit $t_{1}, t_{2} \rightarrow \infty$; daraus folgt: $\left\|x\left(t_{2}\right)-x\left(t_{1}\right)\right\| \rightarrow 0 \quad\left(t_{1}, t_{2} \rightarrow \infty\right) . x(t)$ hat also ein Limeselement, sei es $x^{*}$. Wir zeigen, da $\beta F\left(x^{*}\right)=0$ gilt. Nehmen wir im Gegenteil an, daß $F^{\prime}\left(x^{*}\right) \neq 0$. Infolge der Stetigkeit von $F(x)=\operatorname{grad} \Phi(x)$ gibt es eine $Z$ ahl $\tau>0$, so daß für $t>\tau$ die Ungleichung

$$
\left\|F^{\prime}(x(t))\right\|-\left\|F^{\prime}\left(x^{*}\right)\right\| \leqslant\left\|F^{\prime}(x(t))-F^{\prime}\left(x^{*}\right)\right\| \leqslant \frac{\alpha}{4}\left\|F^{\prime}\left(x^{*}\right)\right\|
$$

gilt. Daraus bekommen wir, indem wir uns auf (i) und (ii) beziehen:

$$
\begin{aligned}
-\left(\dot{x}(t), F^{\prime}\left(x^{*}\right)\right) & =-(\dot{x}(t), F(x(t)))+\left(\dot{x}(t), F^{\prime}(x(t))-F\left(x^{*}\right)\right) \\
& \geqslant \alpha\|\dot{x}(t)\| \cdot\left\|F^{\prime}(x(t))\right\|-\|\dot{x}(t)\| \frac{\alpha}{4}\left\|F^{\prime}\left(x^{*}\right)\right\| \\
& \geqslant \frac{\alpha}{2}\|\dot{x}(t)\| \cdot\left\|F^{\prime}\left(x^{*}\right)\right\| \geqslant \frac{\alpha}{4 \gamma}\left\|F^{\prime}\left(x^{*}\right)\right\|^{2}>0 \quad \text { für } \quad t>\tau .
\end{aligned}
$$

Daraus folgt aber

$$
\begin{aligned}
& \left(x(t)-x(\tau), F\left(x^{*}\right)\right)=\int_{\tau}^{t}\left(\dot{x}(s), F^{F}\left(x^{*}\right)\right) d s \\
& \geqslant \frac{\alpha}{4 \gamma} \int_{\tau}^{t}\left\|F\left(x^{*}\right)\right\|^{2} d s=\frac{a}{4 \gamma}(t-\tau)\left\|F\left(x^{*}\right)\right\|^{2} \rightarrow+\infty \quad(t \rightarrow+\infty),
\end{aligned}
$$

was der bewiesenen Konvergenz $x(t) \rightarrow x^{*}$ widerspricht. Es muß also $F\left(x^{*}\right)=0$ sein, und folglich, laut (iii), auch $\Phi\left(x^{*}\right)=\inf \Phi$, w.z.b.w.

Satz 3 kann gewisse Anwendungen auf das Berechnen von Eigenwerten und Eigenelementen symmetrischer Operationen finden. Es sei $A$ lineare, beschränkte, symmetrische und positive Operation. Wir legen $Z_{1}=\left[x: A x=\lambda_{0} x\right], Z_{2}=H-Z_{1}$ die zu $Z_{1}$ orthogonale komplementäre lineare Menge, $\Phi(x)=(A x, x) /(x, x), \lambda_{0}=\inf [(A x, x) /(x, x)]$ fest, und setzen voraus, daß eine Zahl $\delta>0$ vorhanden ist, so daß die Ungleiehung

$$
(A y, y)-\lambda_{0}(y, y) \geqslant \delta(y, y)
$$

für alle $y \in Z_{2}$ gilt. Unter diesen Voraussetzungen beweisen wir die folgenden Schlüsse:

SchuUss 1. Erfüllt die abstrakte Funktion $x(t)(t \geqslant 0)$ die Gleichung $-d x(t) / d t=-\operatorname{grad} \Phi(x(t))$ und ist $\operatorname{proj}(x(0)) / Z_{1} \neq 0$, so konvergiert $x(t)$ $(t \rightarrow \infty)$ gegen gewisses Element $x^{*}$ und es gilt $A\left(x^{*}\right)=\lambda_{0} x^{*}, \Phi\left(x^{*}\right)=\lambda_{0}$.
Beweis. Für jedes Element $x$ gilt eindeutige orthogonale Zerlegung

$$
x=\xi z+y, \quad z \epsilon Z_{1}, \quad\|z\|=1, \quad y \in Z_{2}
$$

für welche folgende Identitäten leicht nachzuprüfen sind:

$$
\begin{gathered}
\|x\|^{2}=\xi^{2}+\|y\|^{2}, \quad(A x, x)=\lambda_{0} \xi^{2}+(A y, y), \\
\Phi(x)-\inf \Phi=(A y, y)-\lambda_{0}(y, y)\|x\|^{-2}, \\
\operatorname{grad} \Phi(x)=2[(x, x) A x-(A x, x) x]\|x\|^{-4}, \\
\operatorname{grad} \Phi(x), y)=2 \xi^{2}\left[(A y, y)-\lambda_{0}(y, y)\right]\|x\|^{-4} .
\end{gathered}
$$

Aus (9), (7) und (4) bekommen wir die Ungleichung

$$
\|\operatorname{grad} \Phi(x)\| \geqslant 2 \xi^{2} \delta\|x\|^{-3}\left\{\Phi(x)-\inf \Phi^{1 / 2}\right\} .
$$

In der Tat, wir haben:

$\|\operatorname{grad} \Phi(x)\| \geqslant\|y\|^{-1}(\operatorname{grad} \Phi(x), y)$

$$
\begin{aligned}
& =2 \xi^{2}\|y\|^{-1}\left[(A y, y)-\lambda_{0}(y, y)\right]\|x\|^{-4} \\
& =2 \xi^{2}\|y\|^{-1}\left[(A y, y)-\lambda_{0}(y, y)\right]^{1 / 2}\|x\|^{-3}\left[(A y, y)-\lambda_{0}(y, y)\right]^{1 / 2}\|x\|^{-1} \\
& =2 \xi^{2} \delta\|x\|^{-3}(\Phi(x)-\inf \Phi)^{1 / 2}, \quad \text { w.z.b.w. }
\end{aligned}
$$

Es sei nun $x(t)$ eine die Gleichung $d x(t) / d t=-\operatorname{grad} \Phi(x(t))$ erfüllende abstrakte Funktion und $x(t)=\xi(t) z(t)+y(t)$ die entsprechende orthogonale Zerlegung, d.h. $z(t) \in Z_{1},\|z(t)\|=1, y(t) \in Z_{2}$. Gemäß der Voraussetzung des Schlusses gilt: $\xi(0) \neq 0$. Wir beweisen nun, daß $(\xi(t))^{2}$ eine differenzierbare und nichtabnehmende Funktion ist. Wir haben

$$
(\xi(t))^{2}=\|x(t)\|^{2}-\|y(t)\|^{2}=\left\|\operatorname{proj} x(t) \mid Z_{2}\right\|^{2},
$$

so daß sich die Differenzierbarkeit von $(\xi(t))$ als eine Folgerung dieser von $x(t)$ erweist. Da aber

$$
\frac{d}{d t}\|x(t)\|^{2}=2(\dot{x}(t), x(t))=-2(\operatorname{grad} \Phi(x(t)), x(t))=0 ;
$$

weiterhin ist

$$
\begin{aligned}
\frac{d}{d t}(\xi(t))^{2}= & -\frac{d}{d t}\|y(t)\|^{2}=-2,\left(y(t), \frac{d}{d t} y(t)\right)=-2\left(y(t), \frac{d}{d t} \operatorname{proj}(x(t)) \mid Z_{2}\right) \\
= & -2\left(y(t), \operatorname{proj} \dot{x}(t) \mid Z_{2}\right)=2\left(y(t), \operatorname{proj} \operatorname{grad} \Phi(x(t)) \mid Z_{2}\right) \\
= & 4\|x(t)\|^{-4}\left\{\left(y(t),\|x(t)\|^{2} A(y(t))-A(x(t), x(t)) y(t)\right)\right\} \\
= & 4\|x(t)\|^{-4}\left\{(y(t), A(y(t)))\left[(\xi(t))^{2}+\|y(t)\|^{2}\right]-\right. \\
& \left.-\|y(t)\|^{2}\left[\lambda_{0}(\xi(t))^{2}+(A y(t), y(t))\right]\right\} \\
= & 4\|x(t)\|^{-4}(\xi(t))^{2}\left[(A y(t), y(t))-\lambda_{0}\|y(t)\|^{2}\right] \\
\geqslant & =4\|x(t)\|^{-4}\left(\xi(t)^{2}\right) \delta^{2}\|y(t)\|^{2} \geqslant 0,
\end{aligned}
$$


d.h. $(\xi(t))^{2}$ ist wirklich eine nichtabnehmende Funktion. Da außerdem $\|x(t)\|$ eine Konstante ist (wegen $(x(t), \dot{x}(t))=-(x(t), \operatorname{grad} \Phi(x(t)))=0$ ), bekommen wir aus der Ungleichung (10)

$$
\begin{aligned}
\|\operatorname{grad} \Phi(x(t))\| & \geqslant 2 \delta(\xi(t))^{2}\|x(t)\|^{-3} \sqrt{\Phi(x(t))-\inf \Phi} \\
& \geqslant 2 \delta(\xi(0))^{2}\|x(0)\|^{-3}[\Phi(x(t))-\inf \Phi]^{1 / 2} .
\end{aligned}
$$

Die Voraussetzungen des Satzes 3 werden also erfüllt, wenn wir dort $f(s)=2 \delta(\xi(0))^{2}\|x(0)\|^{-3} \sqrt{s}$ einsetzen. Es gibt also ein Element $x^{*}$, für welches $x(t) \rightarrow x^{*}$, grad $\Phi\left(x^{*}\right)=0$ laut $(8)$ :

$\left\|x^{*}\right\|^{2} A\left(x^{*}\right)-\left(A x^{*}, x^{*}\right) x^{*}=0, \quad\left(A x^{*}, x^{*}\right) /\left(x^{*}, x^{*}\right)=\Phi\left(x^{*}\right)=\inf \Phi=\lambda_{0}$, woraus wir auf $A x^{*}=\lambda_{0} x^{*}$ schließen, w.z.b.w.

SchuUss 2. Haben $A, \Phi, \lambda_{0}$ die vorige Bedeutung und ist $x(t)$ eine abstrakte Funktion, die den Voraussetzungen (i), (ii) des Satzes 3 und noch den zusätzlichen

$$
\begin{gathered}
0<C_{1} \leqslant\|x(t)\| \leqslant C_{2} \quad(t \geqslant 0), \\
\Phi(x(0))-\inf \Phi \leqslant \varepsilon^{2}<\delta^{2}
\end{gathered}
$$

genügt, dann konvergiert $x(t)(t \rightarrow \infty)$ gegen ein Element $x$, so daß $A x^{*}=$ $=\lambda_{0} x^{*}$ gilt.

Beweis. Zuerst beweisen wir, daß für jedes $x \in H$ die Ungleichung $\Phi(x)$-inf $\Phi \leqslant \varepsilon^{2}<\delta^{2}$ die Ungleichung

$$
\|\operatorname{grad} \Phi(x)\| \geqslant 2\left(\delta^{2}-\varepsilon^{2}\right) \delta^{-1}\|x\|^{-1}[\Phi(x)-\inf \Phi]^{1 / 2}
$$

nach sich zieht. Tatsächlich, wir haben

woraus

$$
\begin{aligned}
\varepsilon^{2} & \geqslant \Phi(x)-\inf \Phi=\|x\|^{-2}\left[(A y, y)-\lambda_{0}(y, y)\right] \\
& \geqslant\|x\|^{-2} \delta^{2}\|y\|^{2}=\delta^{2}\|x\|^{-2}\left(\|x\|^{2}-\xi^{2}\right)
\end{aligned}
$$

$$
\xi^{2} \geqslant\left(\delta^{2}-\varepsilon^{2}\right) \delta^{-2}\|x\|^{2} ;
$$

diese letzte Ungleichung zusammen mit (10) gibt (13). Ferner bemerken wir, wie im Beweis des Satzes 3 , daß die Funktion $\Phi(x(t))$ niechtwachsend ist, so daß also die Ungleichung $\Phi(x(t))-\inf \Phi$ für $t \geqslant 0$ umsomehr, laut (12) erfüllt ist. Berücksichtigen wir noch (11), so erhalten wir

$$
\|\operatorname{grad} \Phi(x(t))\| \geqslant 2\left(\delta^{2}-\varepsilon^{2}\right) \delta^{-1} C_{2}^{-1}\{\Phi(x(t))-\inf \Phi\}^{1 / 2},
$$

was bedeutet, daß alle Voraussetzungen des Satzes 3 erfüllt sind. Damit ist der Schluß bewiesen.

Anmerkung 1. Es ist leicht eine abstrakte Funktion $x(t)$ herzustellen, die die Voraussetzungen des Satzes 3, sowie die Gleichungen $\|x(t)\|=1, x(0)=a \epsilon H,\|a\|=1$ erfüllt. Zu diesem Zwecke bezeichnen wir kurz $F x=\operatorname{grad} \Phi(x)=2\|x\|^{-4}[(x, x) A x-(A x, x) x]$, und bemerken, daß es zwei positive Zahlen gibt, $M>0, m>0$, so daß für $x, y \in H,\|x\|=$ $=\|y\|=1$, die Ungleichungen $\|F x-F y\| \leqslant m\|x-y\|,\|F x\| \leqslant M$ gelten.

Es sei $\mu$ beliebige Zahl, $0<\mu<1$,

$$
\begin{gathered}
\vartheta=\frac{1}{2}(M+m+M \mu)^{-1}, \\
a_{0}=a, \quad a_{n+1}=\left\|a_{n}-(t-\vartheta) F a_{n}\right\|^{-1}\left(a_{n}-\vartheta F a_{n}\right), \\
x(t)=\left\|a_{n}-(t-n \vartheta) F a_{n}\right\|^{-1}\left(a_{n}-(t-n \vartheta) F a_{n}\right)
\end{gathered}
$$

für $n \vartheta \leqslant t \leqslant(n+1) \vartheta$.

Es sind folgende Identitäten und Ungleichungen leicht nachzuprüfen, indem man $\left(a_{n}, F^{\prime}\left(a_{n}\right)\right)=0$ berücksichtigt:

(14) $\quad x(t)=\frac{d}{d t} x(t)=$

$\left\|a_{n}-(t-n) F a_{n}\right\|\left\{\left\|a_{n}-(t-n \vartheta) F a_{n}\right\|^{2} \cdot F a_{n}-(t-n \vartheta)\left\|F a_{n}\right\|^{2}\left[a_{n}-(t-n \vartheta) \cdot F a_{n}\right]\right\}$,

(15) $\quad\left\|\dot{x}(t)+F a_{n}\right\| \leqslant 2(t-n \vartheta) M\left\|F a_{n}\right\| \leqslant 2 M \vartheta(1-2 \vartheta M)^{-1}\|\dot{x}(t)\|$,

(16) $\quad\left\|x(t)-a_{n}\right\| \leqslant 2(t-n \vartheta)\left\|F a_{n}\right\|$,

(17) $\left\|F(x(t))-F a_{n}\right\| \leqslant 2(t-n \vartheta) \cdot m \cdot\|F a\| \leqslant 2(1-2 \vartheta M)^{-1} \cdot \vartheta m \cdot\|\dot{x}(t)\|$,

(18) $\quad\|\dot{x}(t)+F(x(t))\| \leqslant 2 \vartheta(m+M)(1-2 \vartheta M)^{-1}\|\dot{x}(t)\|=\mu\|\dot{x}(t)\|$.

Aus (18) bekommt man aber nacht einfacher Rechnung:

$$
\begin{aligned}
& (x(t), F(x(t))) \leqslant-\sqrt{\left(1-\mu^{2}\right)}\|\dot{x}(t)\|\|F(x(t))\|, \\
& (1+\mu)^{-1}\|\dot{x}(t)\| \leqslant\|F(x(t))\| \leqslant(1-\mu)^{-1}\|\dot{x}(t)\|,
\end{aligned}
$$

und das sind eben die Voraussetzungen des Satzes 3, wobei

$$
\alpha=\sqrt{\left(1-\mu^{2}\right)}, \quad \beta=(1+\mu)^{-1}, \quad \gamma=(1-\mu)^{-1} .
$$

Ähnliche Betrachtungen liest man bei Kantorovitch [1]; die Methode des schnellsten Falles wurde auch von M. Altman auf numerisches Lösen von Gleichungen angewendet (s. [4], [5], u. [6]). Vergleiche auch die Noten des Autors [7], [8].

\section{Literaturnachweis}

[1] L. Kantorovitch, Über Newtonsche Methode, Trudy Matemat. Instituta im. Steklova 28 (1949), S. 107-144 (russisch).

[2] P. C. Rosenbloom, The method of steepest descent, Proceedings of Symposium in Appl. Math. 6 (1956), S. $127-176$.

[3] F. Riesz et B. S. Nagy, Leçons d'Analyse Fonctionnelle, Budapest 1952. 
[4] M. Altman, On the method of orthogonal projection, Bull. Acad. Polon. Sei. 5 (1957), S. $229-231$.

[5] - An approximative method for solving linear equations in a Hilbert spaces, ibid. 5 (1957), S. 601.

[6] - Connection between the method of steepest descent and a Newton method, ibid. 5 (1957), S. 1031

[7] T. Leżański, Über das Minimumproblem für Funktionale in Banachschen Räumen, Math. Annalen 152 (1963), S. 271-274.

[8] - Eine effektive Lösungsmethode nichtlinearer Gleichungen in Hlibertschen Räumen, ibid. 158 (1965), S. 377-386.

Reçu par la Rédaction le 28. 3. 1966

\section{On simultaneous extension} of infinitely differentiable functions

by

Z. OGRODZKA (Warszawa)

Introduction. The first result concerning the extension of differentiable functions is due to Lichtenstein [8], who has shown that any differentiable function on a closed set in $R^{3}$ whose boundary satisfies some conditions can be extended to a differentiable function in $R^{3}$. Whitney [15] has proved that any function $f(x)=f\left(x_{1}, \ldots, x_{n}\right)$ of class $C^{m}$ on a closed subset of $R^{n}$ can be extended to a function of class $C^{m}$ in $R^{n}$. In the case $m=\infty$ this extension is not linear. Hestenes [6] has modified Whitney's proof, and has given also another method of extending the differentiable functions, which is applicable only when $m$ is finite and the boundary has suitable properties. It is, however, sufficiently general to be of interest and the proof is relatively simple. The method used is a generalization of the reflection principle used by Lichtenstein [8] by the use of McShane's [9] lemma on the extension localisation.

Next, Mitjagin [10], and Ryll-Nardzewski (not published) have shown independently the existence of the linear continuous extension operator $L: C^{\infty}[a, b] \rightarrow C^{\infty}(R)(a<b)$.

Seeley [14] has constructed a linear and continuous extension operator from the space of functions of class $C^{\infty}$ on a half-space to the space of functions of class $C^{\infty}$ on the whole $R^{n}$.

Recently Adams, Aronszajn and Smith (announced in [1]) obtained a complete result concerning the existence of extension, namely a necessary and sufficient condition for a convex domain $D$ to possess the property of extension is that for a certain bounded cone $C$ and for every $x \in D$ there be a congruent cone with a vertex $x$, contained in $\bar{D}$.

The first results concerning the linear extension operators of the continuous functions are contained in the papers of Borsuk [2] and Dugundji [3].

In this paper we prove: (1) the existence of a linear operator extending the functions of class $C^{\infty}$ considered on the closed subset of $R^{n}$ to the functions of class $C^{\infty}$ on the whole $R^{n},(2)$ the existence of a linear operator extending the functions of class $C^{\infty}$ defined on the closed subsets with 